\title{
Significance of Ki-67 in non-muscle invasive bladder cancer patients: a systematic review and meta-analysis
}

\author{
Kyungtae Ko${ }^{1}$, Chang Wook Jeong ${ }^{2}$, Cheol Kwak², Hyeon Hoe Kim² and Ja Hyeon Ku${ }^{2}$ \\ ${ }^{1}$ Department of Urology, Kandong Sacred Heart Hospital, Hallym University College of Medicine, Seoul, Korea \\ ${ }^{2}$ Department of Urology, Seoul National University Hospital, Seoul, Korea \\ Correspondence to: Ja Hyeon Ku, email: kuuro70@snu.ac.kr
}

Keywords: bladder cancer, urothelial carcinoma, Ki-67, prognosis, meta-analysis

Received: August 13, $2017 \quad$ Accepted: September 23, $2017 \quad$ Published: October 13, 2017

Copyright: Ko et al. This is an open-access article distributed under the terms of the Creative Commons Attribution License 3.0 (CC BY

3.0), which permits unrestricted use, distribution, and reproduction in any medium, provided the original author and source are credited.

\section{ABSTRACT}

Purpose: This meta-analysis evaluated the prognostic significance of Ki-67 in non-muscle invasive bladder cancer (NMIBC).

Materials and Methods: We selected 39 articles including 5,229 patients from Embase, Scopus, and PubMed searches. The primary outcomes, recurrence-free survival (RFS), progression-free survival (PFS), disease-specific survival (DSS), and overall survival (OS) were determined using time-to event hazard ratios (HRs) with $\mathbf{9 5 \%}$ confidence intervals (CIs). Study heterogeneity was tested by chi-square and $I^{2}$ statistics. Heterogeneity sources were identified by subgroup meta-regression analysis.

Results: Two studies were prospective; 37 were retrospective. Immunohistochemistry was performed in tissue microarrays or serial sections. A wide range of antibody dilutions and Ki-67 positivity thresholds were used. Study heterogeneity was attributed to analysis results in studies of RFS $(p<0.0001)$. Meta-regression analysis revealed that region and analysis results accounted for heterogeneity in PFS studies $(p=0.00471, p<0.0001)$. High Ki-67 expression was associated with poor RFS (pooled HR, 1.78; 95\% CI, 1.482.15), poor PFS (pooled HR, 1.28; 95\% CI, 1.13-2.15), poor DSS (pooled HR, 2.24; 95\% CI, 1.47-2.15), and worse OS (pooled HR, 2.29; 95\% CI, 1.24-4.22).

Conclusions: The meta-analysis found that current evidence supports the prognostic value of $\mathrm{Ki}-67$ in NMIBC patients.

\section{INTRODUCTION}

Bladder cancer is the ninth most common cancer worldwide. Approximately 430,000 patients are diagnosed and 165,000 patients die from it annually [1]. Approximately $25 \%$ of newly diagnosed cases are muscle invasive bladder cancer (MIBC, $\geq \mathrm{T} 2$ ), and radical cystectomy is the standard treatment. Other non-muscle invasive bladder cancers (NMIBCs) include stage Ta noninvasive papillary carcinomas and stage $\mathrm{T} 1$ tumors that invade the subepithelial connective tissue. The gold standard treatment of NMIBC is transurethral resection of bladder tumor (TURBT) and intravesical Bacillus Calmette-Guérin (BCG) installation. However, 30\%-70\% of patients experience a recurrence after initial treatment, and $25 \%-60 \%$ progress to MIBC.
As the incidence and survival of bladder cancer increase, the importance of treatment follow-up and predicting the risk of recurrence and progression of individual patients also increases. The outcome of $\mathrm{T} 1$ bladder cancer can range from no recurrence to rapid progression to $\mathrm{MIBC}$ and metastasis. As progression has a poor prognosis, it is important to distinguish patients who would benefit from early cystectomy and those best managed by bladder-preserving treatments. Currently, such group assignment is challenging. The use of clinical and pathological variables, such as tumor size and number and presence of a carcinoma in situ (CIS), to estimate MIBC progression risk has been evaluated [2], but it is difficult to estimate individual prognosis. Characterizing bladder cancer as low or high grade using two-tier criteria of the European Treatment Guidelines or the 2004 
World Health Organization classification is difficult, and distinguishing Ta and T1 bladder cancer is problematic because of interobserver error [3]. Tumor markers, such as bcl-2, p53, Ki67, and CK20, are currently under study, but none are in routine clinical use at this time.

$\mathrm{Ki}-67$ is a nuclear protein that is associated with ribosomal RNA transcription and is a marker of cellular proliferation [4]. It is strongly expressed in the growth fraction of cancer cells, and the presence of Ki-67-positive tumor cells indicates a poor survival and recurrence prognosis in prostate and breast cancer and nephroblastoma [5]. Ki-67 has not been confirmed as a poor prognosis marker in NMIBC patients because the reported thresholds of positivity and the immunochemical staining methods vary, making direct comparisons difficult [6]. An expert consensus panel has found that markers, such as Ki-67 and p53, can predict the recurrence and progression of bladder cancer, but the inconsistency of available data indicates their unreliability [7]. This meta-analysis was conducted to increase our understanding of the prognostic significance of Ki-67 in NMIBC patients.

\section{RESULTS}

\section{Study characteristics}

The characteristics of the 39 selected studies are described in Tables 1-3. They were published between 1997 and 2015, 17 were conducted in Asian countries, 17 were conducted in Europe, and five were conducted in America. All but two studies were retrospective, 19 included $<100$ patients, 20 included $\geq 100$ patients, follow-up ranged from 1 to 267 months, and five studies did not report the duration of follow-up.

\section{Immunohistochemistry}

Immunohistochemistry (IHC) was performed using tissue microarrays of 1-2 mm diameter samples of representative tissues and using slide mounted serial tissue sections in the other 34 studies. Fifteen of the 39 studies evaluated IHC staining in formalin-fixed paraffinembedded tissue blocks, but did not identify the primary antibody used, and a wide range of antibody dilutions was reported (1/20 to $1 / 200)$. In 33 studies, immunopositivity was defined by the presence of nuclear staining, but the cutoff percentage for positive or negative expression $(\%$ IHC cutoff) and the reported percentage of Ki-67-positive cells varied widely among studies. Twenty studies reported blinded evaluation of Ki-67 expression (Table 4).

\section{Study outcomes}

Of the 39 studies, the association of Ki-67 expression with recurrence-free survival (RFS) was reported in 34 (4,581 patients), with progression-free survival (PFS) in 21 (3,400 patients), with disease-specific survival (DSS) in six (1,505 patients), and with overall survival (OS) in two (356 patients) studies (Tables 5-8). The most common cofactors included in the multivariate analysis of the risk of outcome were grade and $\mathrm{T}$ stage. Forest plots of the hazard ratios (HRs) reported in individual studies and those from the meta-analysis are shown in Figure 1. Despite the use of strict inclusion criteria, between-study heterogeneity was detected in the effect of Ki-67 expression on RFS and PFS, with $p<0.05$ and $\mathrm{I}^{2} \geq 50 \%$.

\section{RFS}

Overall, the pooled HR for RFS in 34 studies was 1.78 (95\% CI, 1.48-2.15), suggesting that high Ki-67 expression indicated poor bladder cancer prognosis. However, significant heterogeneity was observed in the studies $\left(\mathrm{I}^{2}=80 \%, p<0.00001\right)$ (Figure 1A). Subgroup meta-regression by publication year, region, number of patients, HR estimation, and analysis results identified analysis results as the only possible explanation for heterogeneity ( $p<0.0001$, Table 9$)$. The other variables in the subgroup analyses did not include any heterogeneity of data.

\section{PFS}

A meta-analysis of 21 studies found that high $\mathrm{Ki}$ 67 expression was significantly associated with poor PFS (pooled HR, 1.28; 95\% CI, 1.13-1.44). However, the Cochrane $Q$ test $(p<0.00001)$ and an $\mathrm{I}^{2}=75 \%$ could not exclude significant heterogeneity (Figure 1B). Metaregression analysis revealed that region accounted for part of the study heterogeneity for PFS ( $p=0.00471$, Table $10)$. In addition, analysis results was found to significantly affect the relationship between $\mathrm{Ki}-67$ expression and PFS $(p<0.0001)$. Other variables included in this subgroup analysis did not include any heterogeneity of data.

\section{DSS}

A meta-analysis of six studies found that high $\mathrm{Ki}$ 67 expression was significantly associated with poor DSS (pooled HR, 2.24; 95\% CI, 1.47-3.39). No significant study heterogeneity was found $\left(\mathrm{I}^{2}=0 \%, p=0.73\right.$; Figure $\left.1 \mathrm{C}\right)$.

OS

Meta-analysis of the two studies evaluating the association of ki-67 expression with OS found that a high Ki-67 expression predicted a worse outcome, with a pooled HR of 2.29 (95\% CI, 1.24-4.22). Inter-study heterogeneity was not significant $\left(\mathrm{I}^{2}=12 \%, p=0.29\right)$ (Figure 1D). 


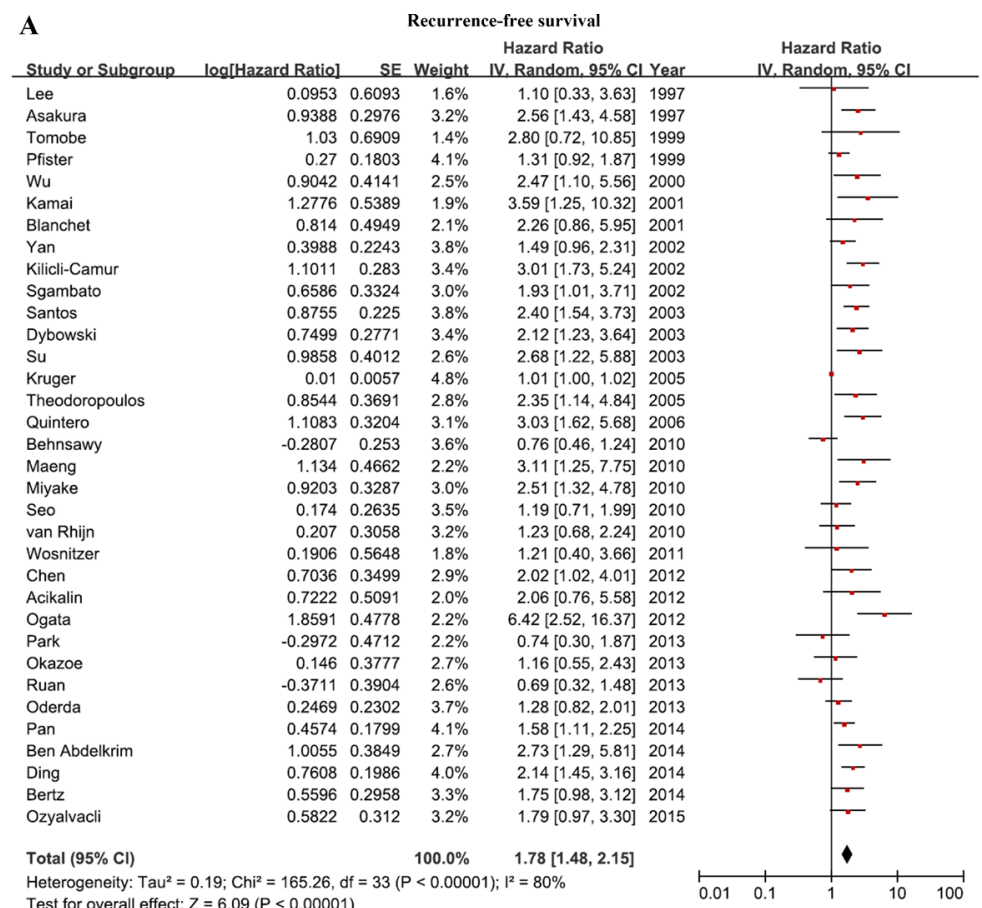

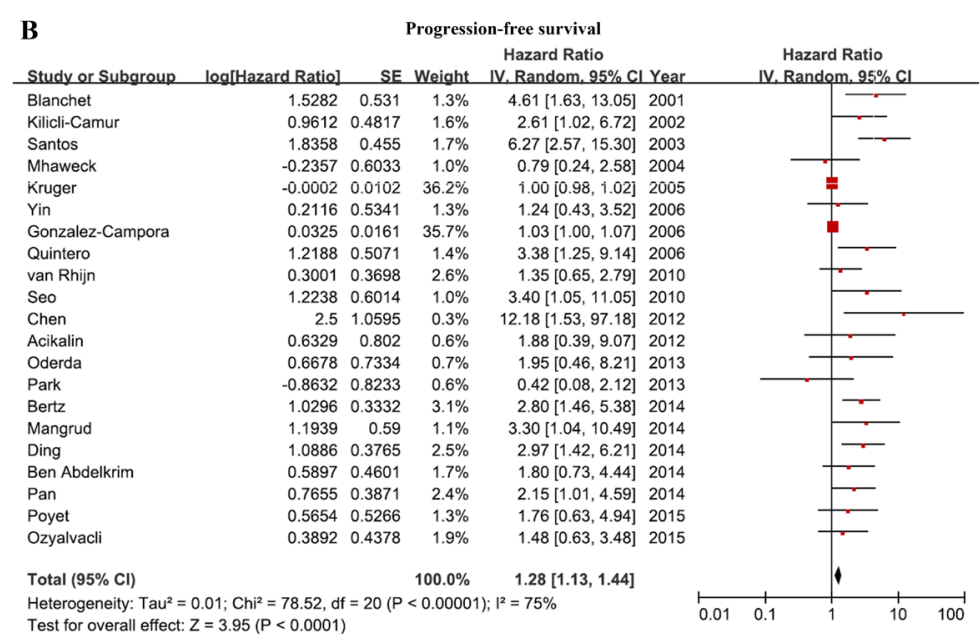
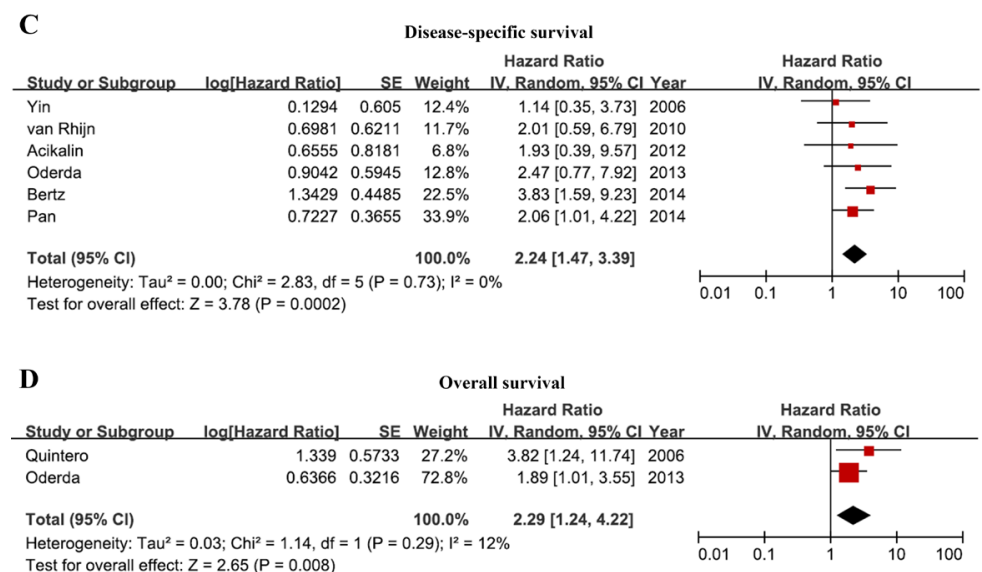

Figure 1: Forest plots of the hazard ratios. High Ki-67 expression indicated poor bladder cancer prognosis. (A) Recurrence-free survival, (B) progression-free survival, (C) disease-specific survival, (D) overall survival. Between-study heterogeneity was detected in the effect of Ki-67 expression on RFS and PFS. 
Table 1: Main characteristics of the eligible studies

\begin{tabular}{|c|c|c|c|c|c|c|c|}
\hline Study & Year & Country & Recruit period & Study design & $\begin{array}{l}\text { Inclusion and } \\
\text { exclusion criteria }\end{array}$ & $\begin{array}{l}\text { Consecutive } \\
\text { patients }\end{array}$ & $\begin{array}{l}\text { Definition of } \\
\text { outcome }\end{array}$ \\
\hline Asakura [20] & 1997 & Japan & 1984-1993 & Retrospective & Yes & NA & No \\
\hline Lee [21] & 1997 & Korea & 1988-1993 & Retrospective & Yes & NA & No \\
\hline Pfister [22] & 1999 & Canada & 1990-1992 & Retrospective & Yes & NA & No \\
\hline Tomobe [23] & 1999 & Japan & 1989-1994 & Retrospective & No & NA & No \\
\hline $\mathrm{Wu}[24]$ & 2000 & Taiwan & 1990-1997 & Retrospective & Yes & NA & No \\
\hline Blanchet [25] & 2001 & France & 1989-1990 & Prospective & No & Yes & Yes \\
\hline Kamai [26] & 2001 & Japan & 1987-1997 & Retrospective & No & Yes & No \\
\hline Kilicli-Camur [27] & 2002 & Turkey & NA & Retrospective & No & NA & Yes \\
\hline Sgambato [28] & 2002 & Italy & 1990-1995 & Retrospective & Yes & Yes & Yes \\
\hline Yan [29] & 2002 & USA & 1994-1999 & Retrospective & Yes & Yes & No \\
\hline Dybowski [30] & 2003 & Poland & 1994-1995 & Retrospective & Yes & NA & No \\
\hline Santos [31] & 2003 & Portugal & 1989-1996 & Retrospective & Yes & Yes & Yes \\
\hline $\mathrm{Su}[32]$ & 2003 & Japan & NA & Retrospective & No & NA & Yes \\
\hline Mhawech [33] & 2004 & Switzerland & $1997-2000$ & Retrospective & Yes & NA & Yes \\
\hline Krüger [34] & 2005 & Germany & 1987-1999 & Retrospective & Yes & Yes & Yes \\
\hline Theodoropoulos [35] & 2005 & Greece & 1993-2003 & Retrospective & Yes & No & Yes \\
\hline Gonzalez-Campora [36] & 2006 & Spain & 1991-1997 & Retrospective & No & Yes & Yes \\
\hline Quintero [37] & 2006 & Spain & 1990-1994 & Retrospective & No & Yes & Yes \\
\hline Yin [38] & 2006 & China & NA & Retrospective & No & Yes & No \\
\hline Maeng [39] & 2010 & Korea & 2001-2007 & Retrospective & No & NA & No \\
\hline Miyake [40] & 2010 & Japan & $2000-2005$ & Retrospective & No & Yes & No \\
\hline Seo $[41]$ & 2010 & Korea & 2001-2007 & Retrospective & Yes & NA & Yes \\
\hline van Rhijn [10] & 2010 & Netherlands & NA & Retrospective & No & NA & Yes \\
\hline Behnsawy [42] & 2011 & Japan & 2000-2007 & Retrospective & No & Yes & No \\
\hline Wosnitzer [43] & 2011 & USA & NA & Retrospective & No & NA & No \\
\hline Acikalin [6] & 2012 & Turkey & $1996-2007$ & Retrospective & No & NA & Yes \\
\hline Chen [11] & 2012 & China & NA & Retrospective & No & NA & Yes \\
\hline Ogata [44] & 2012 & Brazil & 2005-2010 & Retrospective & Yes & NA & No \\
\hline Oderda [45] & 2013 & Italy & 1994-2004 & Prospective & No & NA & Yes \\
\hline Okazoe [46] & 2013 & Japan & 2006-2009 & Retrospective & No & NA & No \\
\hline Park [47] & 2013 & Korea & 1990-2007 & Retrospective & No & NA & Yes \\
\hline Ruan [48] & 2013 & China & $2007-2010$ & Retrospective & Yes & NA & No \\
\hline Ben Abdelkrim [14] & 2014 & Tunisia & 2001-2003 & Retrospective & No & NA & Yes \\
\hline Bertz [18] & 2014 & Germany & 1989-2006 & Retrospective & No & NA & No \\
\hline Ding [15] & 2014 & China & 2000-2010 & Retrospective & No & NA & Yes \\
\hline Mangrud [49] & 2014 & Norway & 2002-2006 & Retrospective & Yes & Yes & Yes \\
\hline Pan [50] & 2014 & Taiwan & 1991-2005 & Retrospective & No & NA & Yes \\
\hline Özyalvaçli [16] & 2015 & Turkey & 2005-2013 & Retrospective & No & Yes & Yes \\
\hline Poyet [17] & 2015 & Switzerland & 1990-2006 & Retrospective & No & Yes & Yes \\
\hline
\end{tabular}

NA: not available.

\section{Sensitivity analysis}

One-way sensitivity analyses were conducted by stepwise exclusion of single studies and recalculating the pooled HR for the remaining studies. No significant differences were observed among the results obtained at each step of the analysis (data not shown), demonstrating that the overall results of the meta-analysis were statistically reliable.

\section{Publication bias}

Because fewer than 10 studies were included in meta-analyses of DSS and OS, it was not reasonable to 
Table 2: Patient characteristics of the eligible studies

\begin{tabular}{|c|c|c|c|c|c|}
\hline Study & $\begin{array}{l}\text { No. of } \\
\text { patients }\end{array}$ & $\begin{array}{l}\text { Median age, range } \\
\text { (years) }\end{array}$ & $\begin{array}{l}\text { Gender } \\
\text { (male/female) }\end{array}$ & Intravesical therapy (no.) & $\begin{array}{l}\text { Median follow-up, range } \\
\text { (months) }\end{array}$ \\
\hline Asakura [20] & 104 & 63 (mean), 28-90 & $78 / 26$ & Chemotherapy (6) & 42 (mean), 3-134 \\
\hline Lee [21] & 32 & NA, $30-81$ & $28 / 4$ & BCG (32) & NA \\
\hline Pfister [22] & 244 & 65.1 (mean), NA & NA & No & 47 (mean), NA \\
\hline Tomobe [23] & 50 & 63.9 (mean), 22-88 & $43 / 7$ & Chemotherapy or BCG (32) & 44 (mean), 5-80 \\
\hline $\mathrm{Wu}[24]$ & 86 & NA & NA & NA & NA \\
\hline Blanchet [25] & 70 & 62.6 (mean), 21-84 & $66 / 4$ & BCG (57) & $64,12-111$ \\
\hline Kamai [26] & 86 & NA & NA & MMC, doxorubicin or BCG (NA) & $50,3-124$ \\
\hline Kilicli-Camur [27] & 118 & 60.2 (mean), 29-86 & NA & NA & 31.4 (mean), 24-60 \\
\hline Sgambato [28] & 96 & 68 (mean), 29-92 & $83 / 13$ & BCG (NA) & 50 (mean), 24-102 \\
\hline Yan [29] & 270 & 71 (mean), NA & $\begin{array}{l}\text { 196/71, } \\
\text { unknown (3) }\end{array}$ & BCG (66) & $19,(1-54)$ \\
\hline Dybowski [30] & 45 & NA & NA & NA & $64,1-82$ \\
\hline Santos [31] & 159 & $66,21-88$ & $115 / 44$ & Chemotherapy (65), BCG (17) & $46.5,4-123$ \\
\hline $\mathrm{Su}[32]$ & 79 & $64,34-91$ & $66 / 13$ & MMC or Adriamycin (74) & 48.7 (mean), 4-78 \\
\hline Mhawech [33] & 49 & 70.3 (mean), 52-90 & $44 / 5$ & BCG (7) & $12,3-77$ \\
\hline Krüger [34] & 73 & 68 , NA & $60 / 13$ & BCG (73) & NA \\
\hline Theodoropoulos [35] & 140 & $69,23-89$ & $107 / 33$ & Epirubicin or BCG (114) & $41,8-131$ \\
\hline Gonzalez-Campora [36] & 147 & 66 (mean), 30-95 & $127 / 20$ & BCG (NA) & 75 (mean), 5-12 yr \\
\hline Quintero [37] & 164 & 61 (mean), 29-93 & $143 / 21$ & BCG (NA) & $75,60-144$ \\
\hline Yin [38] & 101 & NA & $81 / 20$ & BCG (101) & $\begin{array}{l}54,20-68.6 \\
(10-90 \% \text { percentiles })\end{array}$ \\
\hline Maeng [39] & 55 & 67 (mean), 33-84 & $40 / 15$ & NA & 26.2 (mean), 3-70 \\
\hline Miyake [40] & 109 & 68.5 (mea), 36-94 & $19 / 14$ & $\begin{array}{l}\text { Anthracycline (16), doxorubicin } \\
\text { (1), epirubicin (13), pirarubicin } \\
\text { (2), BCG (19) }\end{array}$ & $48,1-99$ \\
\hline Seo [41] & 129 & $64.2(38-88)$ & $104 / 25$ & MMC (129) & 48.6 (mean), 6.1-96 \\
\hline van Rhijn [10] & 230 & 65.1 (mean), NA & $175 / 55$ & NA & 8.6 yr, 6.6-11.3 yr (IQR) \\
\hline Behnsawy [42] & 161 & NA & $137 / 24$ & Unknown regimen (49) & $47,13-93$ \\
\hline Wosnitzer [43] & 32 & $70.3,44-89$ & $25 / 7$ & $\begin{array}{l}\text { Docetaxel (17), nanoparticle } \\
\text { albumin-bound docetaxel (15) }\end{array}$ & $22,11-75$ \\
\hline Acikalin [6] & 68 & $63,35-85$ & $66 / 2$ & NA & $51,12-132$ \\
\hline Chen [11] & 72 & 61.3 (mean), 27-87 & $58 / 14$ & $\begin{array}{l}\text { MMC, epirubicin, pirarubicin } \\
\text { (NA) }\end{array}$ & 63.4 (mean), 16-93 \\
\hline Ogata [44] & 43 & $70,39-85$ & $35 / 8$ & $\mathrm{NA}$ & NA, $12-71$ \\
\hline Oderda [45] & 192 & 73.2 (mean), NA & $166 / 26$ & BCG (192) & $100,2-229$ \\
\hline Okazoe [46] & 71 & $72,41-95$ & $59 / 12$ & Unknown regimen (31) & $9.8,1.0-51.8$ \\
\hline Park [47] & 70 & $66,31-85$ & $53 / 8$ & BCG (70) & $60,6-217$ \\
\hline Ruan [48] & 126 & 64.5 (mean), 29-90 & $103 / 23$ & NA & NA \\
\hline Ben Abdelkrim [14] & 71 & 63.1 (mean), 39-88 & $67 / 4$ & NA & $28,3-77$ \\
\hline Bertz [18] & 309 & $71.7,38-87$ & $237 / 72$ & BCG (309) & $49,5-172$ \\
\hline Ding [15] & 332 & $67,21-92$ & $273 / 59$ & NA & $47,2-124$ \\
\hline Mangrud [49] & 193 & $74,39-95$ & $148 / 45$ & BCG (NA) & $75,1-127$ \\
\hline Pan $[50]$ & 605 & 71 (mean), 23-92 & $511 / 94$ & $\begin{array}{l}\text { MMC (272), doxorubicin (67), } \\
\text { epirubicin (130), BCG (132) }\end{array}$ & NA \\
\hline Özyalvaçli [16] & 90 & NA & $83 / 7$ & NA & $32.8,36.2-103.6(\mathrm{IQR})$ \\
\hline Poyet [17] & 158 & $69.5,32-92$ & $131 / 43$ & NA & $110.6,32.4-266.8$ \\
\hline
\end{tabular}

NA: not available, BCG: bacille Calmette-Guérin, MMC: mitomycin C, IQR: interquartile range. 
estimate the potential for publication bias. No obvious asymmetry was evident in any of the funnel plots shown in Figure 2. The $p$-values of the Begg tests for RFS and PFS were $>0.05$ ( $p=0.4676$ for RFS and 0.4324 for PFS), which confirmed the funnel plot symmetry and lack of evidence of publication bias.

\section{DISCUSSION}

About $75 \%$ of newly diagnosed bladder cancers are NMIBC localized in the subepithelial connective tissue [8]. After initial TURBT, NMIBC patients undergo cystoscopy every 3 months for the first year to monitor recurrence and progression. This protocol is painful and is also a financial burden; however, because progression to MIBC has a bad prognosis for the patients, ongoing cystoscopy and radiological evaluation are required. Early cystectomy for high risk T1 bladder cancer patients who are expected to progress is important because it can increase survival. On the other hand, radical cystectomy is a surgical procedure with many complications and requires use of urostomy bags or clean intermittent catheterizations, both of which have negative effects on

daily activities. Efforts to distinguish candidates for early cystectomy or bladder preservation are complicated by the heterogeneous clinical behavior of bladder cancer.

Until recently, predicting the progression from NMIBC to MIBC has relied on clinicopathological variables, such as tumor size, grade, multiplicity, and diagnosis of CIS. However, even in cases of the same stage and grade of NMIBC, the clinical course can vary from no recurrence to rapid progression, making it difficult to predict the course. In addition, inter-pathologist variation in interpretation of TURBT specimens can occur because of malorientation, cautery artifacts, and other reasons. Given the current situation, reliable molecular markers would assist in making clinical decisions.

Previous studies of tumorigenesis indicated that changes at the molecular level precede changes in cellular morphology [9]. Changes in gene expression in multiple molecular pathways have been related to the development of bladder cancer. $\mathrm{Ki}-67$ has been associated with expression of oncogenes or tumor suppressor genes, such as Connexin 43, Sox2, G protein-coupled receptor 87 , heme oxygenase-1, p53, and p27 [17, 26, 37, 40, 46, 48]. IHC assays of proliferation markers, such as the Ki-
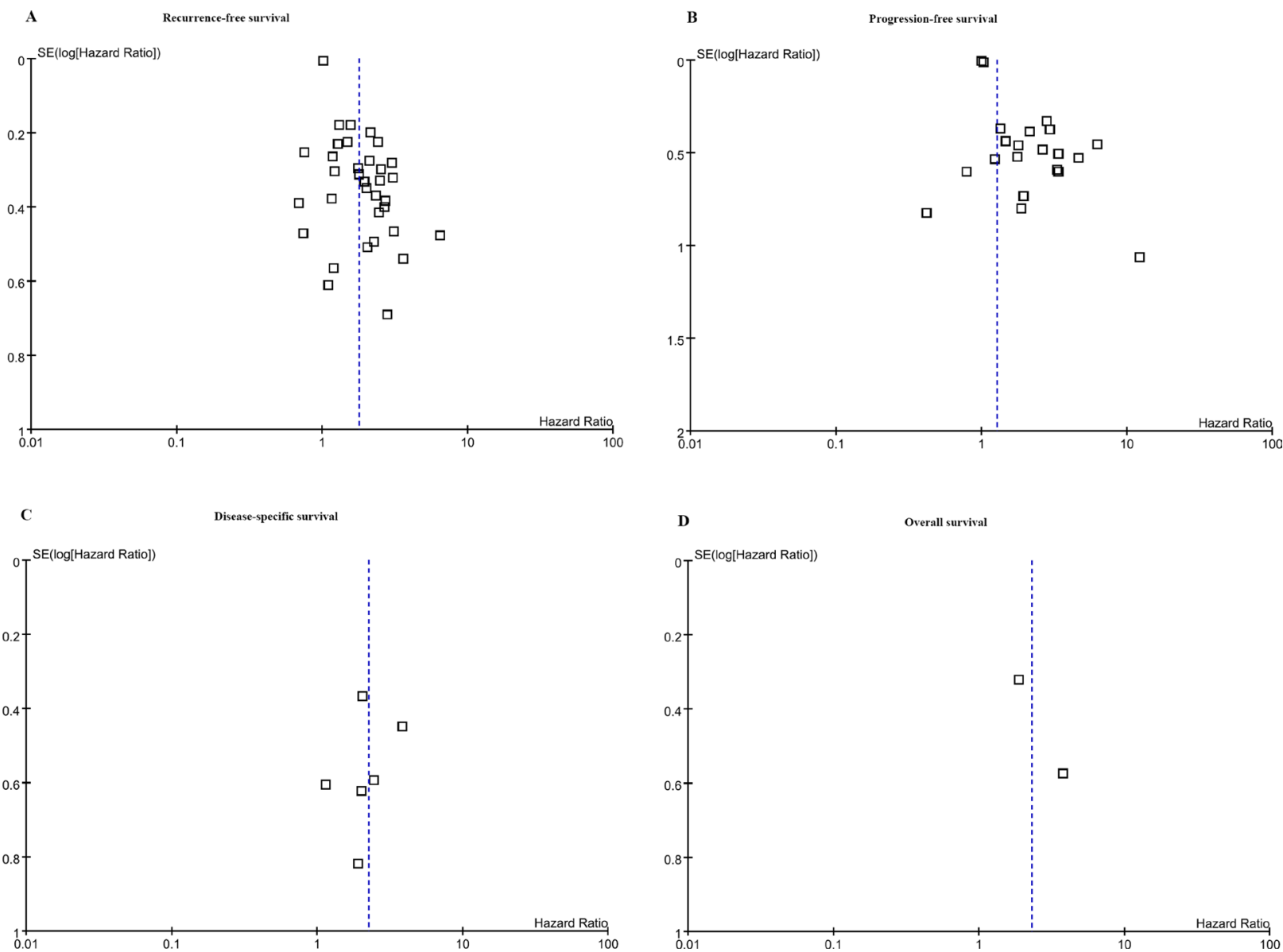

Figure 2: Begg tests for (A) recurrence-free survival and (B) progression-free survival confirmed the funnel plot symmetry and lack of evidence of publication bias. Fewer than 10 studies were included in meta-analyses of (C) disease-specific survival and (D) overall survival. 
Table 3: Tumor characteristics of the eligible studies

\begin{tabular}{|c|c|c|c|c|c|c|c|c|c|c|c|c|c|c|c|c|}
\hline \multirow[t]{2}{*}{ Study } & \multicolumn{3}{|c|}{ T stage } & \multicolumn{3}{|c|}{ Grade } & \multicolumn{2}{|c|}{ Concomitant CIS } & \multicolumn{2}{|c|}{ Multiplicity } & \multicolumn{2}{|c|}{ Size } & \multicolumn{2}{|c|}{ Tumor architecture } & \multicolumn{2}{|c|}{ History } \\
\hline & Tis & Ta & T1 & G1 & G2 & G3 & Absent & Present & Single & Multiple & $<3 \mathrm{~cm}$ & $\geq 3 \mathrm{~cm}$ & Papillary & Non-papillary & Primary & Recurrent \\
\hline Asakura [20] & & 61 & 43 & 30 & 63 & 11 & NA & NA & NA & NA & $\mathrm{NA}$ & NA & NA & NA & 104 & NA \\
\hline Lee [21] & 0 & 0 & 42 & 0 & 16 & 16 & 30 & 2 & 42 & 0 & NA & NA & 26 & 6 & 17 & 15 \\
\hline Pfister [22] & 0 & 194 & 50 & 83 & NA & NA & NA & NA & 163 & 81 & 152 & 92 & NA & NA & 244 & 0 \\
\hline Tomobe [23] & 0 & 6 & 44 & 15 & 28 & 7 & NA & NA & 26 & 24 & NA & NA & NA & NA & 34 & 16 \\
\hline $\mathrm{Wu}[24]$ & NA & NA & NA & NA & NA & 0 & NA & NA & 86 & 0 & NA & NA & 86 & 0 & 86 & 0 \\
\hline Blanchet [25] & 0 & 43 & 27 & 12 & 25 & 33 & 63 & 7 & 30 & 17 & NA & NA & $\mathrm{NA}$ & NA & 70 & 0 \\
\hline Kamai [26] & NA & NA & NA & NA & NA & NA & NA & NA & NA & NA & NA & NA & NA & NA & NA & NA \\
\hline Kilicli-Camur [27] & 0 & 59 & 59 & 45 & 51 & 22 & NA & NA & NA & NA & NA & NA & NA & NA & 60 & 58 \\
\hline Sgambato [28] & 0 & 42 & 54 & 13 & 51 & 32 & NA & NA & 96 & 0 & NA & NA & NA & NA & 96 & 0 \\
\hline Yan [29] & 0 & 215 & 55 & 57 & 183 & 30 & 270 & 0 & NA & NA & NA & NA & NA & NA & NA & NA \\
\hline Dybowski [30] & 0 & 25 & 20 & NA & NA & NA & 45 & 0 & NA & NA & NA & NA & NA & NA & NA & NA \\
\hline Santos [31] & 0 & 56 & 103 & 61 & 98 & 0 & 159 & 0 & 122 & 37 & NA & NA & NA & NA & 159 & 0 \\
\hline $\mathrm{Su}[32]$ & 0 & 33 & 46 & 23 & 56 & 0 & NA & NA & 43 & 36 & 65 & 14 & 56 & 23 & 79 & 0 \\
\hline Mhawech [33] & 0 & 0 & 49 & 0 & 38 & 11 & NA & NA & 30 & 19 & NA & NA & NA & NA & 49 & 0 \\
\hline Krüger [34] & 0 & 0 & 73 & 0 & 33 & 40 & NA & NA & 27 & 46 & NA & NA & NA & NA & 73 & 0 \\
\hline Theodoropoulos [35] & 0 & 42 & 98 & 30 & 88 & 22 & NA & NA & NA & NA & NA & NA & NA & NA & 140 & 0 \\
\hline Gonzalez-Campora [36] & 0 & 63 & 84 & 29 & 92 & 26 & NA & NA & NA & NA & 57 & 90 & NA & NA & 147 & 0 \\
\hline Quintero [37]" & 0 & 80 & 84 & 31 & 92 & 41 & NA & NA & NA & NA & 109 & 55 & NA & NA & 164 & 0 \\
\hline Yin [38]" & 0 & 54 & 47 & 0 & 59 & 42 & 101 & 0 & NA & NA & NA & NA & NA & NA & NA & $\mathrm{NA}$ \\
\hline Maeng [39]" & 0 & 38 & 17 & 10 & 22 & 23 & NA & NA & 44 & 11 & 35 & 20 & 50 & 5 & 33 & 22 \\
\hline Miyake [40] & 2 & 24 & 83 & 9 & 74 & 26 & 98 & 11 & 54 & 55 & 87 & 22 & NA & NA & 109 & 0 \\
\hline Seo [41] & 0 & 81 & 46 & 31 & 76 & 22 & 129 & 0 & 36 & 84 & 60 & 57 & 104 & 15 & 101 & 28 \\
\hline van Rhijn [10] & 0 & 171 & 59 & 88 & 108 & 34 & 218 & 12 & 165 & 65 & NA & NA & NA & NA & 230 & 0 \\
\hline Behnsawy [42] & 0 & 65 & 25 & 29 & 49 & 12 & 76 & 14 & 46 & 44 & 72 & 18 & 80 & 10 & 90 & 0 \\
\hline Wosnitzer [43]" & 9 & 5 & 18 & 0 & 0 & 32 & 24 & 8 & NA & NA & NA & NA & NA & NA & 0 & 32 \\
\hline Acikalin [6] & 0 & 0 & 68 & 11 & 31 & 26 & NA & NA & 23 & 45 & 16 & 52 & NA & NA & NA & NA \\
\hline Chen [11]" & 0 & 19 & 53 & 16 & 38 & 18 & NA & NA & 49 & 23 & 43 & 29 & NA & NA & NA & NA \\
\hline Ogata [44]" & 0 & 41 & 2 & 0 & 26 & 14 & 43 & 0 & 43 & 0 & 24 & 19 & 43 & 0 & 43 & 0 \\
\hline Oderda [45] & 0 & 121 & 115 & 53 & 76 & 63 & 182 & 10 & 58 & 134 & 159 & 31 & NA & NA & 113 & 79 \\
\hline Okazoe $[46]^{*}$ & 2 & 53 & 16 & 0 & 46 & 25 & NA & NA & 34 & 37 & 54 & 12 & 58 & 13 & 44 & 27 \\
\hline Park [47] & 0 & 0 & 61 & 0 & 0 & 61 & 56 & 5 & 23 & 38 & 36 & 25 & 38 & 23 & 61 & 0 \\
\hline Ruan [48] & 0 & 0 & 126 & NA & NA & 55 & 126 & 0 & 75 & 51 & NA & NA & NA & NA & NA & NA \\
\hline Ben Abdelkrim [14] & 0 & 39 & 32 & 26 & 35 & 10 & NA & NA & NA & NA & NA & NA & NA & NA & 71 & 0 \\
\hline Bertz [18] & 0 & 0 & 309 & 0 & 89 & 220 & 202 & 106 & 106 & 203 & 128 & 181 & 257 & 52 & NA & NA \\
\hline Ding [15] & 0 & 204 & 128 & 114 & 168 & 50 & 309 & 23 & NA & NA & 221 & 111 & NA & NA & NA & NA \\
\hline Mangrud [49] & 0 & 154 & 39 & 44 & 98 & 51 & 171 & 22 & 92 & 73 & NA & NA & NA & NA & 193 & 0 \\
\hline $\operatorname{Pan}[50]^{*}$ & 0 & 336 & 231 & 38 & 256 & 311 & NA & NA & NA & NA & NA & NA & NA & NA & NA & NA \\
\hline Özyalvaçli [16]* & 0 & 41 & 49 & 0 & 45 & 45 & NA & NA & 53 & 37 & 46 & 43 & NA & NA & 90 & 0 \\
\hline Poyet [17] & 0 & 90 & 68 & 44 & 86 & 28 & 12 & 146 & 115 & 43 & $\mathrm{NA}$ & NA & 151 & 7 & 158 & 0 \\
\hline
\end{tabular}

"Grading according to the 2004 WHO classification system: papillary urothelial neoplasm of low malignant potential, low grade and high grade.

CIS: carcinoma in situ, NA: not available.

67 and fibroblast growth factor receptor (FGFR)-3 are available in most pathology laboratories and have high reproducibility $[10,11]$. IHC is currently used worldwide by over $90 \%$ of pathologists to diagnose bladder cancer, and $\mathrm{Ki}-67$ is already used as a prognostic marker in over $84 \%$ of specimens in Europe [12]. Another advantage of this biologic marker is that objective measurements are possible and changes in expression can be compared after the therapeutic intervention.

Despite many advantages, biologic markers are not widely used to make clinical decisions because difficulties in making direct comparisons of study results have resulted in lack of consensus on their usefulness. In this meta-analysis, the overexpression threshold varied from $5 \%$ to $25 \%$ and the variation in positive $\mathrm{Ki}-67$ expression was from $10 \%$ to 70 percent. Reasons for the inconsistency of previous study results include different follow-up protocols after TURBT, and differences in patient ethnicity, geography, tumor stage, tissue sectioning methods, and the primary antibodies and antibody dilutions used in each study [6]. The importance of these differences was apparent in the inter-study heterogeneity 
Table 4: Immunohistochemical analysis of the eligible studies

\begin{tabular}{|c|c|c|c|c|c|c|c|c|}
\hline Study & Tissue section & $\begin{array}{l}\text { Primary } \\
\text { antibody }\end{array}$ & Dilution & Compartment & $\begin{array}{l}\text { Definition of } \\
\text { ki-67 index }\end{array}$ & $\begin{array}{l}\% \text { IHC } \\
\text { cut-off }\end{array}$ & $\begin{array}{l}\% \mathrm{ki}-67 \\
\text { positive }\end{array}$ & Interpretation \\
\hline Asakura [20] & All specimens & $\mathrm{NA}$ & $1: 200$ & Nuclei & Yes & 5.35 & 50 & NA \\
\hline Lee [21] & All specimens & NA & NA & Nuclei & Yes & 16 & 50 & Blind \\
\hline Pfister [22] & All specimens & Monoclonal & $1: 50$ & Nuclei & No & 10 & 70 & Blind \\
\hline Tomobe [23] & All specimens & NA & $1: 200$ & Nuclei & Yes & 15.5 & 50 & NA \\
\hline $\mathrm{Wu}[24]$ & All specimens & NA & $1: 100$ & Nuclei & Yes & 10.9 & 50 & Blind \\
\hline Blanchet [25] & All specimens & Monoclonal & $\mathrm{NA}$ & NA & Yes & 13 & 18.5 & Blind \\
\hline Kamai [26] & All specimens & Monoclonal & NA & Nuclei & Yes & 30 & 18.6 & NA \\
\hline Kilicli-Camur [27] & All specimens & Monoclonal & $1: 30$ & Nuclei & Yes & 25 & NA & NA \\
\hline Sgambato [28] & All specimens & Monoclonal & $1: 100$ & Nuclei & Yes & 10 & 65.6 & Blind \\
\hline Yan [29] & All specimens & NA & NA & Nuclei & No & 25 & 34.2 & NA \\
\hline Dybowski [30] & All specimens & Monoclonal & $1: 50$ & Nuclei & No & 30 & 50 & Blind \\
\hline Santos [31] & All specimens & NA & $1: 50$ & Nuclei & Yes & 18 & 50 & NA \\
\hline $\mathrm{Su}[32]$ & All specimens & NA & $1: 50$ & Nuclei & Yes & 18 & 50 & NA \\
\hline Mhawech [33] & $\begin{array}{l}\mathrm{TM} \\
\text { (1.6 mm core) }\end{array}$ & NA & $1: 50$ & Nuclei & Yes & NA & 50 & Blind \\
\hline Krüger [34] & $\mathrm{TM}(2 \times 2 \mathrm{~mm})$ & Monoclonal & $1: 20$ & Nuclei & Yes & Continuous & - & Blind \\
\hline Theodoropoulos [35] & All specimens & NA & Prediluted & Nuclei & Yes & 8.6 & 50 & Blind \\
\hline Gonzalez-Campora [36] & All specimens & Monoclonal & $1: 20$ & Nuclei & Yes & 10 & 18.4 & NA \\
\hline Quintero [37] & All specimens & Monoclonal & Prediluted & Nuclei & Yes & 13 & 10.4 & NA \\
\hline Yin [38] & All specimens & Monoclonal & $1: 100$ & Nuclei & Yes & 20 & 24.8 & NA \\
\hline Maeng [39] & All specimens & NA & $1: 80$ & Nuclei & Yes & 25 & 36.4 & NA \\
\hline Miyake [40] & All specimens & Monoclonal & Prediluted & Nuclei & Yes & 25 & 40.4 & Blind \\
\hline Seo [41] & All specimens & Monoclonal & $1: 50$ & Nuclei & Yes & 25 & 36.4 & NA \\
\hline van Rhijn [10] & All specimens & NA & NA & NA & NA & 25 & NA & Blind \\
\hline Behnsawy [42] & All specimens & Monoclonal & $1: 200$ & Nuclei & Yes & 5 & 28.6 & Blind \\
\hline Wosnitzer [43] & All specimens & Monoclonal & NA & NA & Yes & 10 & 50 & Blind \\
\hline Acikalin [6] & All specimens & Monoclonal & $1: 50$ & Nuclei & Yes & 10 & 69.1 & Blind \\
\hline Chen [11] & All specimens & Monoclonal & $1: 50$ & Nuclei & Yes & 25 & 47.2 & NA \\
\hline Ogata [44] & All specimens & Monoclonal & $1: 100$ & NA & No & 20 & 58.1 & NA \\
\hline Oderda [45] & All specimens & Monoclonal & $1: 10$ & Nuclei & Yes & 20 & NA & NA \\
\hline Okazoe [46] & All specimens & Monoclonal & $1: 100$ & Nuclei & Yes & 18 & 29.6 & Blind \\
\hline Park [47] & $\begin{array}{l}\mathrm{TM} \\
(1 \mathrm{~mm} \text { core })\end{array}$ & Monoclonal & $1: 200$ & Nuclei & Yes & 10.4 & 40 & Blind \\
\hline Ruan [48] & All specimens & Polyclonal & $1: 50$ & Nuclei & Yes & 10 & 55.6 & Blind \\
\hline Ben Abdelkrim [14] & All specimens & NA & $1: 50$ & Nuclei & Yes & 10 & 38 & Blind \\
\hline Bertz [18] & All specimens & Monoclonal & $1: 50$ & Nuclei & Yes & 15 & 64.4 & NA \\
\hline Ding [15] & All specimens & Monoclonal & $1: 100$ & Nuclei & No & 25 & 32.5 & NA \\
\hline Mangrud [49] & All specimens & NA & NA & NA & Yes & 39 & 25 & NA \\
\hline Pan [50] & $\begin{array}{l}\mathrm{TM} \\
(2 \mathrm{~mm} \text { core })\end{array}$ & NA & $1: 100$ & Nuclei & Yes & $20 / 80$ & NA & Blind \\
\hline Özyalvaçli [16] & All specimens & Monoclonal & NA & Nuclei & Yes & 10 & 27.8 & Blind \\
\hline Poyet [17] & $\begin{array}{l}\text { TM } \\
\text { (1 mm core })\end{array}$ & NA & $1: 50$ & NA & Yes & 10 & 38.4 & NA \\
\hline
\end{tabular}

IHC: immunohistochemistry, NA: not available, TM: tissue microarray.

detected in the meta-analysis, with $\mathrm{I}^{2}$ values of $80 \%$ in RFS and $75 \%$ in PFS. To the best of our knowledge, this was the first meta-analysis of Ki-67 in bladder cancer. To determine the origins of the heterogeneity, we performed a meta-regression including publication year, region, HR estimation, and analysis results. Only analysis results were significantly associated with heterogeneity of studies reporting RFS. Although region might have accounted for 
Table 5: Estimation of the hazard ratio for recurrence-free survival

\begin{tabular}{|c|c|c|c|c|}
\hline Study & Analysis & HR estimation & Co-factors & Analysis results \\
\hline Asakura [20] & Multivariate & $\mathrm{HR}, 95 \% \mathrm{CI}$ & T stage, grade, multiplicity, size & Significant \\
\hline Lee [21] & Multivariate & $\mathrm{HR}, 95 \% \mathrm{CI}$ & P53, bcl-2, cathepsin-D & Not significant \\
\hline Pfister [22] & Multivariate & $\mathrm{HR}, 95 \% \mathrm{CI}$ & $\begin{array}{l}\text { T stage, grade, multiplicity, size, } \mathrm{p} 53 \text {, } \\
\text { MDM2, p21 }\end{array}$ & Not significant \\
\hline Tomobe [23] & Multivariate & $\mathrm{HR}, p$ value & $\begin{array}{l}\text { T stage, grade, multiplicity, size, recurrence } \\
\text { history, whole NOR, proliferating NOR, } \\
\text { resting NOR }\end{array}$ & Not significant \\
\hline $\mathrm{Wu}[24]$ & Multivariate & $\mathrm{HR}, 95 \% \mathrm{CI}$ & T stage, grade, $\mathrm{p} 53$, bcl- 2 & Significant \\
\hline Blanchet [25] & Univariate & Event no., $P$ value & - & Not significant \\
\hline Kamai [26] & Multivariate & $\mathrm{HR}, 95 \% \mathrm{CI}$ & Grade, p27, cyclin E & Significant \\
\hline Kilicli-Camur [27] & Univariate & Event no., $P$ value & - & Significant \\
\hline Sgambato [28] & Multivariate & $\mathrm{HR}, 95 \% \mathrm{CI}$ & Age, T stage, grade, p27, cyclin D1 & Significant \\
\hline Yan [29] & Multivariate & $\mathrm{HR}, 95 \% \mathrm{CI}$ & T stage, p53 & Not significant \\
\hline Dybowski [30] & Univariate & Event no., $P$ value & - & Significant \\
\hline Santos [31] & Multivariate & $\mathrm{HR}, 95 \% \mathrm{CI}$ & T stage, grade, multiplicity, BCG, p53 & Significant \\
\hline $\mathrm{Su}[32]$ & Multivariate & $\mathrm{HR}, 95 \% \mathrm{CI}$ & T stage, tumor architecture, $\mathrm{p} 53$, c-erbB-2 & Significant \\
\hline Krüger [34] & Multivariate & $\mathrm{HR}, 95 \% \mathrm{CI}$ & Grade, p53 & Not significant \\
\hline Theodoropoulos [35] & Multivariate & $\mathrm{HR}, 95 \% \mathrm{CI}$ & $\begin{array}{l}\text { T stage, grade, apoptotic index, p53, bcl-2, } \\
\text { VEGF, MVD, HIF- } 1 \alpha\end{array}$ & Significant \\
\hline Quintero [37] & Multivariate & $\mathrm{HR}, 95 \% \mathrm{CI}$ & Size & Significant \\
\hline Maeng [39] & Univariate & $\mathrm{HR}, 95 \% \mathrm{CI}$ & - & Significant \\
\hline Miyake [40] & Multivariate & $\mathrm{HR}, 95 \% \mathrm{CI}$ & Grade, $\mathrm{p} 53, \mathrm{HO}-1$ & Significant \\
\hline Seo $[41]$ & Univariate & $\mathrm{HR}, 95 \% \mathrm{CI}$ & - & Not significant \\
\hline van Rhijn [10] & Multivariate & $\mathrm{HR}, 95 \% \mathrm{CI}$ & $\begin{array}{l}\text { Age, sex, hospital, T stage, grade, } \\
\text { concomitant CIS, multiplicity, size, EORTC } \\
\text { risk score, FGFR3 }\end{array}$ & Not significant \\
\hline Behnsawy [42] & Univariate & $\mathrm{HR}, 95 \% \mathrm{CI}$ & - & Not significant \\
\hline Wosnitzer [43] & Multivariate & $\mathrm{HR}, 95 \% \mathrm{CI}$ & $\begin{array}{l}\text { Age, sex, T stage, concomitant CIS, p53, } \\
\text { stathmin, tau }\end{array}$ & Not significant \\
\hline Acikalin [6] & Multivariate & $\mathrm{HR}, 95 \% \mathrm{CI}$ & Age, grade, size, multiplicity, mapsin & Not significant \\
\hline Chen [11] & Multivariate & $\mathrm{HR}, 95 \% \mathrm{CI}$ & $\begin{array}{l}\text { Age, sex, T stage, grade, multiplicity, size, } \\
\text { intravesical instillation, VEGF }\end{array}$ & Significant \\
\hline Ogata [44] & Univariate & Event no., $P$ value & - & Significant \\
\hline Oderda [45] & Multivariate & $\mathrm{HR}, 95 \% \mathrm{CI}$ & Age, T stage, grade, ,multiplicity, size, p53 & Not significant \\
\hline Okazoe [46] & Univariate & $\mathrm{HR}, 95 \% \mathrm{CI}$ & - & Not significant \\
\hline Park [47] & Multivariate & $\mathrm{HR}, 95 \% \mathrm{CI}$ & p53, pRb, PTEN, p27, FGFR3, CD9 & Not significant \\
\hline Ruan [48] & Multivariate & $\mathrm{HR}, 95 \% \mathrm{CI}$ & Age, sex, grade, multiplicity, size, Sox 2 & Significant \\
\hline Ben Abdelkrim [14] & Univariate & Event no., $P$ value & - & Significant \\
\hline Bertz [18] & Multivariate & $\mathrm{HR}, 95 \% \mathrm{CI}$ & $\begin{array}{l}\text { Age, sex, grade, concomitant CIS, tumor } \\
\text { architecture, p53, CK20 }\end{array}$ & Not significant \\
\hline Ding [15] & Multivariate & $\mathrm{HR}, 95 \% \mathrm{CI}$ & $\begin{array}{l}\text { T stage, grade, concomitan CIS, } \\
\text { multiplicity, size }\end{array}$ & Significant \\
\hline Pan $[50]$ & Multivariate & $\mathrm{HR}, 95 \% \mathrm{CI}$ & $\begin{array}{l}\text { T stage, grade, multiplicity, size, } \\
\text { intravesical instillation, p53, HSP27, COX2, } \\
\text { cyclin D1, p16, pRb, p27, p21, EGFR, } \\
\text { E-cadherin, EpCam, no. of altered markers }\end{array}$ & Significant \\
\hline Özyalvaçli [16] & Multivariate & $\mathrm{HR}, 95 \% \mathrm{CI}$ & T stage, smoking, size, $\mathrm{P} 16 \mathrm{~d}$ & Not significant \\
\hline
\end{tabular}

HR: hazard ratio, CI: confidence interval, NOR: nucleolar organizer regions, BCG: bacille Calmette-Guérin, VEGF: vascular endothelial growth factor, MVD, microvessel density, HIF: hypoxia-inducible factor, CIS: carcinoma in situ, EORTC: European Organization for Research and Treatment of Cancer, EGFR: epithelial growth factor receptor. 
Table 6: Estimation of the hazard ratio for progression-free survival

\begin{tabular}{|c|c|c|c|c|}
\hline Study & Analysis & HR estimation & Co-factors & Analysis results \\
\hline Blanchet [25] & Multivariate & $\mathrm{HR}, 95 \% \mathrm{CI}$ & T state, grade, concomitant CIS, multiplicity, size & Significant \\
\hline Kilicli-Camur [27] & Univariate & Event no., $P$ value & - & Significant \\
\hline Santos [31] & Multivariate & $\mathrm{HR}, 95 \% \mathrm{CI}$ & T stage, grade, multiplicity. BCG, p53 & Significant \\
\hline Mhawech [33] & Multivariate & $\mathrm{HR}, 95 \% \mathrm{CI}$ & P53, p21, cyclin D1, p27, p16 & Not significant \\
\hline Krüger [34] & Univariate & $\mathrm{HR}, 95 \% \mathrm{CI}$ & - & Not significant \\
\hline Gonzalez-Campora [36] & Multivariate & $\mathrm{HR}, 95 \% \mathrm{CI}$ & NA & Significant \\
\hline Quintero [37] & Multivariate & $\mathrm{HR}, 95 \% \mathrm{CI}$ & None & Significant \\
\hline Yin [38] & Multivariate & $\mathrm{HR}, 95 \% \mathrm{CI}$ & $\begin{array}{l}\text { Age, T stage, grade, BIRC5-cytoplasmic labeling index, } \\
\text { BIRC5-nuclear labeling index }\end{array}$ & Not significant \\
\hline Seo $[41]$ & Multivariate & $\mathrm{HR}, 95 \% \mathrm{CI}$ & T stage, grade, tumor architecture, lymphovascular invasion & Significant \\
\hline van Rhijn [10] & Multivariate & $\mathrm{HR}, 95 \% \mathrm{CI}$ & $\begin{array}{l}\text { Age, sex, hospital, T stage, grade, concomitant CIS, } \\
\text { multiplicity, size, EORTC risk score, } F G F R 3\end{array}$ & Not significant \\
\hline Acikalin [6] & Multivariate & $\mathrm{HR}, 95 \% \mathrm{CI}$ & Age, grade, size, multiplicity, mapsin & Not significant \\
\hline Chen [11] & Multivariate & $\mathrm{HR}, 95 \% \mathrm{CI}$ & $\begin{array}{l}\text { Age, sex, T stage, grade, multiplicity, size, intravesical } \\
\text { instillation, VEGF }\end{array}$ & Significant \\
\hline Oderda [45] & Multivariate & $\mathrm{HR}, 95 \% \mathrm{CI}$ & Age, T stage, grade, ,multiplicity, size, p53 & Not significant \\
\hline Park [47] & Multivariate & $\mathrm{HR}, 95 \% \mathrm{CI}$ & p53, pRb, PTEN, p27, FGFR3, CD9 & Not significant \\
\hline Ben Abdelkrim [14] & Univariate & Event no., $P$ value & - & Not significant \\
\hline Bertz [18] & Multivariate & $\mathrm{HR}, 95 \% \mathrm{CI}$ & $\begin{array}{l}\text { Age, sex, grade, concomitant CIS, tumor architecture, p53, } \\
\text { CK20 }\end{array}$ & Significant \\
\hline Ding [15] & Multivariate & $\mathrm{HR}, 95 \% \mathrm{CI}$ & T stage, grade, concomitan CIS, multiplicity, size & Significant \\
\hline Mangrud [49] & Univariate & $\mathrm{HR}, 95 \% \mathrm{CI}$ & - & Significant \\
\hline Pan $[50]$ & Multivariate & $\mathrm{HR}, 95 \% \mathrm{CI}$ & $\begin{array}{l}\text { T stage, grade, multiplicity, size, intravesical instillation, } \\
\text { p53, HSP27, COX2, cyclin D1, p16, pRb, p27, p21, EGFR, } \\
\text { E-cadherin, EpCam, no. of altered markers }\end{array}$ & Significant \\
\hline Özyalvaçli [16] & Univariate & Event no., $P$ value & - & Not significant \\
\hline Poyet [17] & Multivariate & $\mathrm{HR}, 95 \% \mathrm{CI}$ & Grade, tumor architecture, $\mathrm{Cx} 43$ & Not significant \\
\hline
\end{tabular}

HR: hazard ratio, CI: confidence interval, CIS: carcinoma in situ, BCG: bacille Calmette-Guérin, NA: not available, EORTC: European Organization for Research and Treatment of Cancer, VEGF: vascular endothelial growth factor, EGFR: epithelial growth factor receptor.

\section{Table 7: Estimation of the hazard ratio for disease-specific survival}

\begin{tabular}{|c|c|c|c|c|}
\hline Study & Analysis & HR estimation & Co-factors & Analysis results \\
\hline Yin [38] & Multivariate & $\mathrm{HR}, 95 \% \mathrm{CI}$ & $\begin{array}{l}\text { Age, T stage, grade, BIRC5-cytoplasmic } \\
\text { labeling index, , BIRC5-nuclear labeling } \\
\text { index }\end{array}$ & Not significant \\
\hline van Rhijn [10] & Multivariate & $\mathrm{HR}, 95 \% \mathrm{CI}$ & $\begin{array}{l}\text { Age, sex, hospital, T stage, grade, concomitant } \\
\text { CIS, multiplicity, size, EORTC risk score, } \\
\text { FGFR3 }\end{array}$ & Not significant \\
\hline Acikalin [6] & Univariate & Event no., $P$ value & - & Not significant \\
\hline Oderda [45] & Multivariate & $\mathrm{HR}, 95 \% \mathrm{CI}$ & Age, T stage, grade, ,multiplicity, size, p53 & Not significant \\
\hline Bertz [18] & Multivariate & $\mathrm{HR}, 95 \% \mathrm{CI}$ & $\begin{array}{l}\text { Age, sex, grade, concomitant CIS, tumor } \\
\text { architecture, p53, CK20 }\end{array}$ & Significant \\
\hline Pan $[50]$ & Multivariate & $\mathrm{HR}, 95 \% \mathrm{CI}$ & $\begin{array}{l}\text { T stage, grade, multiplicity, size, intravesical } \\
\text { instillation, p53, HSP27, COX2, cyclin D1, } \\
\text { p16, pRb, p27, p21, EGFR, E-cadherin, } \\
\text { EpCam, no. of altered markers }\end{array}$ & Significant \\
\hline
\end{tabular}

HR: hazard ratio, CI: confidence interval, CIS: carcinoma in situ, EORTC: European Organization for Research and Treatment of Cancer, EGFR: epithelial growth factor receptor. 
Table 8: Estimation of the hazard ratio for overall survival

\begin{tabular}{lllll}
\hline Study & Analysis & HR estimation & Co-factors & Analysis results \\
\hline Quintero [37] & Multivariate & HR, 95\% CI & Size, p27 & Significant \\
Oderda [45] & Multivariate & HR, 95\% CI & Age, T stage, grade, ,multiplicity, size, p53 & Significant \\
\hline
\end{tabular}

HR: hazard ratio, CI: confidence interval.

Table 9: Subgroup analysis for recurrence-free survival

\begin{tabular}{llccccc}
\hline & $\begin{array}{l}\text { No. of included } \\
\text { articles }\end{array}$ & No. of cases & Pooled HR (95\% CI) & $\mathbf{C h i}^{2}(\boldsymbol{p}$ value) & $\mathbf{I}^{2}$ & $\boldsymbol{P}_{\mathbf{h}}{ }^{*}$ \\
\hline $\begin{array}{l}\text { Publication year } \\
\text { 1997-2009 }\end{array}$ & 16 & 1,816 & $2.05(1.52-2.76)$ & $92.96(<0.00001)$ & $84 \%$ & 0.1633 \\
$2010-2015$ & 18 & 2,765 & $1.58(1.26-1.96)$ & $37.18(0.003)$ & $54 \%$ & \\
Region & & & & & 0.7686 \\
Asia & 16 & 2,167 & $1.66(1.29-2.13)$ & $33.06(0.005)$ & $55 \%$ & \\
Europe & 14 & 1,825 & $1.91(1.41-2.58)$ & $76.87(<0.00001)$ & $83 \%$ & \\
America & 4 & 589 & $1.81(1.04-3.15)$ & $9.93(0.02)$ & $70 \%$ & \\
No. of patients & & & & & & 0.3895 \\
$<100$ & 18 & 1,189 & $1.95(1.44-2.65)$ & $69.11(<0.00001)$ & $75 \%$ & \\
$\geq 100$ & 16 & 3,392 & $1.66(1.36-2.03)$ & $37.44(0.001)$ & $60 \%$ & \\
HR estimation & & & & & & 0.5542 \\
Univariate & 9 & 763 & $1.99(1.30-3.05)$ & $29.03(0.0003)$ & $72 \%$ & \\
Multivariate & 25 & 3,818 & $1.72(1.40-2.12)$ & $111.81(<0.00001)$ & $79 \%$ & \\
Analysis results & & & & & & $<0.0001$ \\
Not significant & 16 & 2,091 & $1.22(1.05-1.43)$ & $22.48(0.10)$ & $33 \%$ & \\
Significant & 18 & 2,490 & $2.28(1.93-2.70)$ & $22.27(0.17)$ & $24 \%$ & \\
\hline
\end{tabular}

HR: hazard ratio, CI: confidence interval.

$\mathrm{P}_{\mathrm{h}}{ }^{*}$ for heterogeneity between subgroups with meta-regression analysis.

part of the inter-study heterogeneity, analysis results was observed to significantly affect the relationship of Ki-67 expression and PFS.

As a proliferation-associated nuclear antigen, $\mathrm{Ki}$ 67 is expressed in all phases of the cell cycle except $\mathrm{G}_{0}$. The normal bladder uroephithelium has a very low proliferation rate [13], increased proliferation may signal recurrence rate, and high $\mathrm{Ki}-67$ expression has a poor prognosis for patients with bladder cancer. Bladder tumors with Ki-67 expression have aggressive behaviors, such as multifocality, concomitant CIS, and increased EORCT risk scores, in addition to higher grade/stage $[14,15]$. Because Ki-67 is a cellular proliferation marker, some studies claim that it is more closely related to the recurrence of NMIBC rather than progression to MIBC $[14,16]$. Other studies reported that $\mathrm{Ki}-67$ was related not only to recurrence but also to progression and survival $[15,17,18]$. Even though a consensus on the prognosis of Ki-67 expression has not been reached, this meta-analysis found that patients with high Ki-67 expression had significantly higher recurrence and progression rates than those with low expression. Even though the meta-analysis of DSS included only six studies and that of OS only two, patients with high Ki-67 expression had a significantly worse prognosis.

There were two notable study limitations. The first was study heterogeneity, which is common to metaanalyses of prognostic marker studies. Even though we applied strict inclusion and exclusion criteria to all study stages, and the selected studies included patient populations with similar T stage and grade, the variables evaluated study was different and diverse. Second, because of the strict selection criteria, we were not able to perform Begg tests as fewer than 10 studies were included in the DSS and OS meta-analysis. Consequently, while the analysis generated symmetrical inverted funnel plots, the results should be interpreted with care because of publication bias.

\section{MATERIALS AND METHODS}

This meta-analysis was performed following the Preferred Reporting Items for Systematic Reviews and Meta-Analyses (PRISMA) guidelines [19]. 
Table 10: Subgroup analysis for progression-free survival

\begin{tabular}{|c|c|c|c|c|c|c|}
\hline & No. of included articles & No. of cases & Pooled HR (95\% CI) & $\mathrm{Chi}^{2}$ ( $p$ value $)$ & $\mathbf{I}^{2}$ & $\boldsymbol{P}_{\mathrm{h}}^{*}$ \\
\hline Publication year & & & & & & 0.1633 \\
\hline 1997-2009 & 8 & 881 & $1.08(0.97-1.19)$ & $37.11(<0.00001)$ & $81 \%$ & \\
\hline 2010-2015 & 13 & 2,519 & $2.11(1.62-2.75)$ & $11.71(0.47)$ & $0 \%$ & \\
\hline Region & & & & & & 0.0471 \\
\hline Asia & 6 & 1,309 & $2.16(1.19-3.93)$ & $8.96(0.11)$ & $44 \%$ & \\
\hline Europe & 15 & 2,091 & $1.17(1.05-1.30)$ & $55.75(<0.00001)$ & $75 \%$ & \\
\hline No. of patients & & & & & & 0.2529 \\
\hline$<100$ & 8 & 563 & $1.53(0.91-2.59)$ & $18.15(0.01)$ & $61 \%$ & \\
\hline$\geq 100$ & 13 & 2,837 & $2.26(1.50-3.43)$ & $54.85(<0.00001)$ & $78 \%$ & \\
\hline HR estimation & & & & & & 0.418 \\
\hline Univariate & 5 & 545 & $1.61(0.97-2.69)$ & $10.50(0.03)$ & $62 \%$ & \\
\hline Multivariate & 16 & 2,855 & $2.11(1.41-3.15)$ & $62.59(<0.00001)$ & $76 \%$ & \\
\hline Analysis results & & & & & & $<0.0001$ \\
\hline Not significant & 10 & 1,102 & $1.00(0.98-1.02)$ & $7.10(0.63)$ & $0 \%$ & \\
\hline Significant & 11 & 2,298 & $3.02(1769-5.21)$ & $66.75(<0.00001)$ & $85 \%$ & \\
\hline
\end{tabular}

HR: hazard ratio, CI: confidence interval, NMIBC: non-muscle invasive bladder cancer.

$\mathrm{P}_{\mathrm{h}}{ }^{*}$ for heterogeneity between subgroups with meta-regression analysis.

\section{Search strategy}

Embase, Scopus, and PubMed were searched for articles published in English to March 28, 2016 using the keywords "bladder cancer" and "Ki-67." The titles and abstracts of the retrieved articles were reviewed independently by two authors (KK and $\mathrm{CWJ}$ ) to minimize bias and to improve reliability. The reference lists of the retrieved articles were manually searched for potentially eligible studies that were not included in the initial database search. The full texts of the selected articles were independently screened by the same authors. Disagreements between the reviewers were resolved by consensus.

\section{Study selection}

The PRISMA flow chart of the systematic literature search and study selection is shown in Figure 3. The initial searches retrieved 1,959 articles. Of these, 1,059 were excluded as duplicate publications and an additional 575 were excluded after reviewing the abstracts. The full texts of the remaining 325 articles were reviewed, and an additional 286 articles that did not satisfy the inclusion criteria were excluded. A total of 39 articles including 5,229 patients, ranging from 32 to 605 per study were finally included in the analysis [ 6 , $10,11,14-18,20-50]$.

\section{Inclusion and exclusion criteria}

Following the PRISMA guidelines, the study population, intervention, comparator, outcome, and study design (PICOS) were used to define study eligibility [19]. In this analysis, these were defined as Population, patients with NMIBC; Intervention: TURBT; Comparator, Ki-67 expression; Outcome, recurrence, progression, cancerspecific mortality, and any-cause mortality; Study design, univariate and/or multivariate Cox regression analysis. Strict, well-defined inclusion and exclusion criteria were intended to limit heterogeneity across studies and facilitate obtaining clinically meaningful results in this metaanalysis of prognostic marker studies [51]. The eligibility criteria were as follows: publication as an original article in English language; included human research subjects who were NMIBC patients and treated with TURBT; reported the histologic type as urothelial carcinoma (UC); evaluated Ki-67 expression in bladder cancer tissue by IHC; and investigated the association of Ki-67 expression level and survival outcomes. Eligible articles reported Kaplan-Meier/Cox regression-derived results of the prognostic value of Ki-67 on outcomes following the REporting recommendations for tumor MARKer prognostic studies (REMARK) guidelines for assessment of prognostic markers [52].

Studies were excluded if they were: letters, commentaries, case reports, reviews, or conference 
abstracts (because of limited data); studies conducted in animals or cell lines; studies using other than survival analyses.

If the same patient series was included in more than one publication, only the most informative or complete report was included to avoid duplication of the survival data. Two reviewers (CK and HHK) independently determined study eligibility. Discrepant opinions were resolved by discussion.

\section{End points}

The primary outcome measures were RFS, PFS, DSS, and OS. Survival was defined as the time from TURBT to the last follow-up. In the meta-analysis, recurrence was the development of histologically confirmed UC on follow-up after complete tumor resection. Disease-specific death was any death because of bladder cancer in patients with documented metastatic or recurrent disease. Compared with the primary tumor, progression was defined in individual studies as development of a higher stage $[6,14]$; development of a higher stage and/or grade [27,31]; development of a higher stage and/or grade as well as development of regional or distant metastases [25]; development of a higher stage or metastasis [10, 16, 17, 33, 36, 37, 41], or development of a higher stage and muscle invasive cancer ( $\geq \mathrm{T} 2)$, distant metastasis, or death from bladder cancer [11]. Additional definitions of progression included development of MIBC
( $\geq$ T2) $[34,45,47]$ and development of MIBC ( $\geq$ T2) and/ or metastasis $[15,49,50]$.

\section{Data extraction}

Two reviewers (KK and JHK) extracted the study characteristics and outcome data, which were subsequently crosschecked to ensure their accuracy. Any discrepancies in extracting data were resolved by discussion. Authors of eligible studies were not contacted for additional data. The data retrieved following the REMARK guidelines were: the name of first author, country and year of publication, geographic location, study design, and recruitment period; the study population sample size, mean or median age, gender distribution, inclusion and exclusion criteria, treatment administered, endpoint definition, and follow-up period; tumor characteristics including stage, and grade; IHC data including cutoff value of positive expression, the antibodies used; adoption of a blinded evaluation method; and statistical data including survival curves, data including the total number of case and control participants, and HRs with confidence intervals (CIs). Discrepancies were resolved by discussion.

\section{Statistical analysis}

The meta-analysis was carried out with Review Manager software (RevMan 5; The Nordic Cochrane Center, The Cochrane Collaboration, Copenhagen,

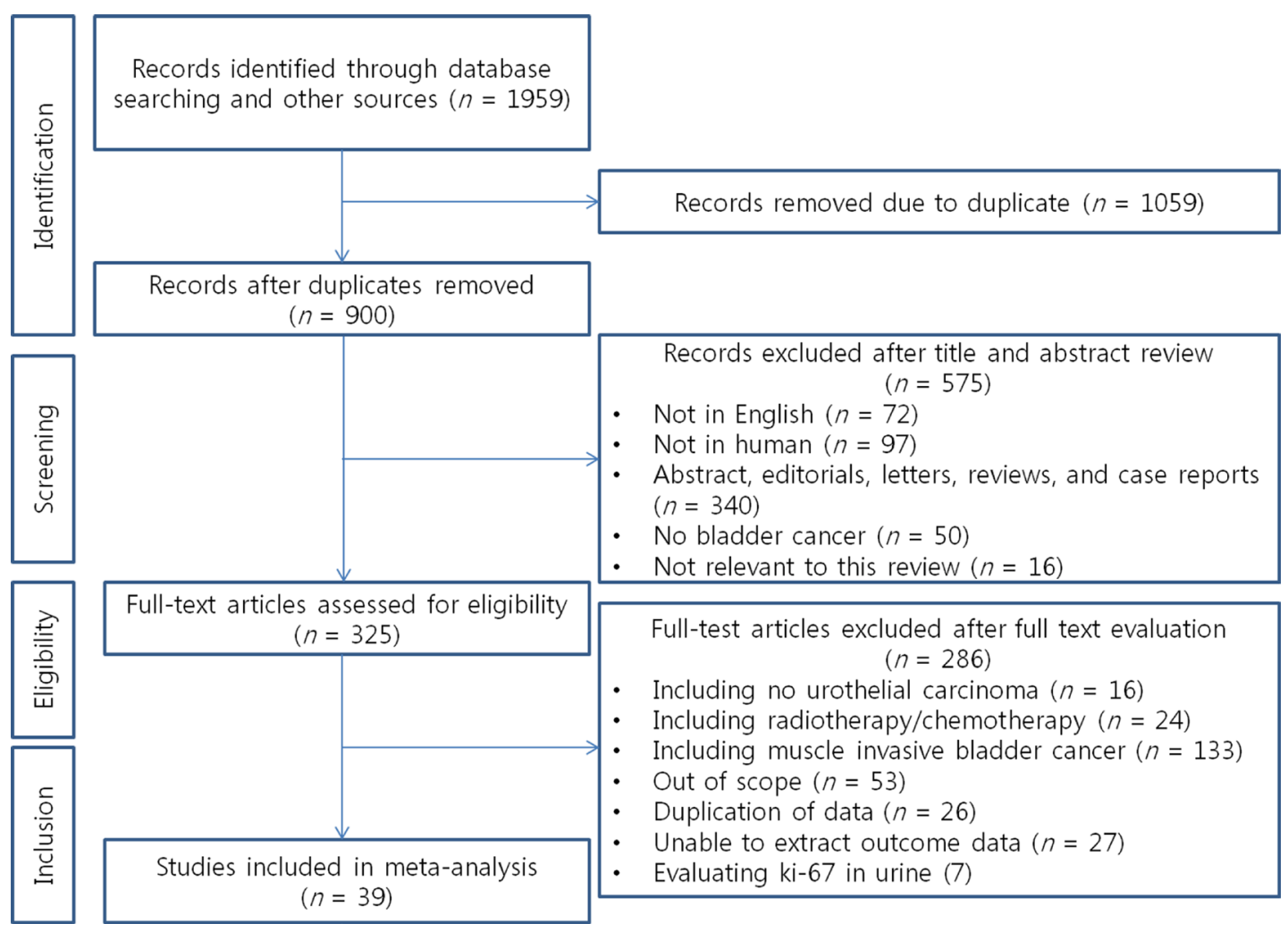

Figure 3: The PRISMA flow chart. 
Denmark) and R 2.13.0 (R Development Core Team, Vienna, Austria, http://www.R-project.org).

\section{Primary analysis}

Study and pooled estimates were presented as forest plots. Survival outcome data were synthesized using the time-to-event HR as the operational measure. The method used to estimate the HR of each publication depended on the data provided. If HRs and the corresponding standard errors were not directly reported, then previously reported indirect methods were used to extract the $\log \mathrm{HR}$ and variance because of the lack of previously published prognostic values [53-55]. A DerSimonian and Laird random effects model was used to obtain the summary HRs and 95\% CIs.

\section{Assessment of heterogeneity}

Heterogeneity of combined HRs was evaluated by the chi-square test and Higgins I-squared statistic. With the chi-square test, heterogeneity was significant when the $p$-value was $<0.05 . \mathrm{I}^{2}$ described the proportion of total variation in meta-analysis estimates that was caused by inter-study heterogeneity, rather than sampling error. It can take a value from $0 \%$ to $100 \%$; increasing $\mathrm{I}^{2}$ values indicated increasing between-study heterogeneity. An $\mathrm{I}^{2}$ value above $50 \%$ was considered as having notable heterogeneity [56, 57], and if found, a subgroup metaregression analysis was carried out to identify the source of the heterogeneity.

\section{Publication bias}

Publication bias was evaluated with funnel plots. In the absence of bias, the plots should resemble a symmetrical, inverted funnel and in the presence of bias, they should appear skewed and asymmetrical [57]. If more than 10 studies were included in the meta-analysis, then the Begg rank correlation test was also used to evaluate publication bias [58]. Bias was assumed if the $p$-value was $<0.05$.

\section{Role of the funding source}

The funding source had no role in the study design, the collection, analysis, and interpretation of data, or the writing of the report. The corresponding author had full access to all data and had final responsibility to submit the paper for publication.

\section{Abbreviations}

MIBC: Muscle invasive bladder cancer; NMIBC: Non-muscle invasive bladder cancer; TURBT: Transurethral resection of bladder tumorl CIS: Carcinoma in situ; IHC: Immunohistochemistry; RFS: Recurrencefree survival; PFS: Progression-free survival; DSS: Disease-specific survival; OS: Overall survival; HRs: Hazard ratios; PRISMA: Preferred Reporting Items for Systematic Reviews and Meta-Analyses; PICOS: Population, intervention, comparator, outcome, and study; UC: Urothelial carcinoma; REMARK: REporting recommendations for tumor MARKer prognostic studies; CIs: Confidence intervals.

\section{Author contributions}

Kyungtae Ko: Drafting of the manuscript, Acquisition of data, analysis and interpretation of data, Change Wook Jeong: Acquisition of data, Cheol Kwak: Analysis of data: Hyeon Hoe Kim; Analysis of data: Ja Hyeon Ku; Analysis and Interpretation of data, Statistical Analysis, Obtainin funding.

\section{CONFLICTS OF INTEREST}

The authors declare no competing financial interests.

\section{FUNDING}

This study was supported by the National Research Foundation of Korea (NRF) grant funded by the Korea government (MSIP) (No. 2016R1A2B4011623).

\section{REFERENCES}

1. GLOBOCAN 2012 v1.0, cancer incidence and mortality worldwide: IARC CancerBase No. 11. International Agency for Research on Cancer. http://globocan.iarc.fr.

2. Sylvester RJ, van der Meijden AP, Oosterlinck W, Witjes JA, Bouffioux C, Denis L, Newling DW, Kurth K. Predicting recurrence and progression in individual patients with stage Ta T1 bladder cancer using EORTC risk tables: a combined analysis of 2596 patients from seven EORTC trials. Eur Urol. 2006; 49:466-5; discussion 75-7. https:// doi.org/10.1016/j.eururo.2005.12.031.

3. Comperat E, Egevad L, Lopez-Beltran A, Camparo P, Algaba F, Amin M, Epstein JI, Hamberg H, Hulsbergen-van de Kaa C, Kristiansen G, Montironi R, Pan CC, Heloir F, et al. An interobserver reproducibility study on invasiveness of bladder cancer using virtual microscopy and heatmaps. Histopathology. 2013; 63:756-66. https://doi.org/10.1111/ his. 12214.

4. Bullwinkel J, Baron-Luhr B, Ludemann A, Wohlenberg C, Gerdes J, Scholzen T. Ki-67 protein is associated with ribosomal RNA transcription in quiescent and proliferating cells. J Cell Physiol. 2006; 206:624-35. https://doi. org/10.1002/jcp.20494.

5. Scholzen T, Gerdes J. The Ki-67 protein: from the known and the unknown. J Cell Physiol. 2000; 182:311-22. https:// 
doi.org/10.1002/(SICI)1097-4652(200003)182:3<311::AIDJCP1>3.0.CO;2-9.

6. Acikalin D, Oner U, Can C, Acikalin MF, Colak E. Predictive value of maspin and $\mathrm{Ki}-67$ expression in transurethral resection specimens in patients with $\mathrm{T} 1$ bladder cancer. Tumori. 2012; 98:344-50. https://doi. org/10.1700/1125.12403.

7. Habuchi T, Marberger M, Droller MJ, Hemstreet GP 3rd, Grossman HB, Schalken JA, Schmitz-Drager BJ, Murphy WM, Bono AV, Goebell P, Getzenberg RH, Hautmann SH, Messing E, et al. Prognostic markers for bladder cancer: International Consensus Panel on bladder tumor markers. Urology. 2005; 66:64-74. https://doi.org/10.1016/j. urology.2005.08.065.

8. Montironi R, Lopez-Beltran A. The 2004 WHO classification of bladder tumors: a summary and commentary. Int J Surg Pathol. 2005; 13:143-53. https:// doi.org/10.1177/106689690501300203.

9. Kausch I, Bohle A. Molecular aspects of bladder cancer III. Prognostic markers of bladder cancer. Eur Urol. 2002; 41:15-29.

10. van Rhijn BW, Zuiverloon TC, Vis AN, Radvanyi F, van Leenders GJ, Ooms BC, Kirkels WJ, Lockwood GA, Boeve ER, Jobsis AC, Zwarthoff EC, van der Kwast TH. Molecular grade (FGFR3/MIB-1) and EORTC risk scores are predictive in primary non-muscle-invasive bladder cancer. Eur Urol. 2010; 58:433-41. https://doi. org/10.1016/j.eururo.2010.05.043.

11. Chen JX, Deng N, Chen X, Chen LW, Qiu SP, Li XF, Li JP. A novel molecular grading model: combination of Ki67 and VEGF in predicting tumor recurrence and progression in non-invasive urothelial bladder cancer. Asian Pac J Cancer Prev. 2012; 13:2229-34.

12. Lopez-Beltran A, Algaba F, Berney DM, Boccon-Gibod L, Camparo P, Griffiths D, Mikuz G, Montironi R, Varma M, Egevad L. Handling and reporting of transurethral resection specimens of the bladder in Europe: a web-based survey by the European Network of Uropathology (ENUP). Histopathology. 2011; 58:579-85. https://doi.org/10.1111/ j.1365-2559.2011.03784.x.

13. Sarkis AS, Dalbagni G, Cordon-Cardo C, Zhang ZF, Sheinfeld J, Fair WR, Herr HW, Reuter VE. Nuclear overexpression of $\mathrm{p} 53$ protein in transitional cell bladder carcinoma: a marker for disease progression. J Natl Cancer Inst. 1993; 85:53-9.

14. Ben Abdelkrim S, Rammeh S, Ziadi S, Tlili T, Jaidane M, Mokni M. Expression of topoisomerase II alpha, ki67, and p53 in primary non-muscle-invasive urothelial bladder carcinoma. J Immunoassay Immunochem. 2014; 35:35867. https://doi.org/10.1080/15321819.2014.899254.

15. Ding W, Gou Y, Sun C, Xia G, Wang H, Chen Z, Tan J, $\mathrm{Xu}$ K, Qiang D. Ki-67 is an independent indicator in nonmuscle invasive bladder cancer (NMIBC); combination of EORTC risk scores and Ki-67 expression could improve the risk stratification of NMIBC. Urol Oncol. 2014; 32:42 e13-9. https://doi.org/10.1016/j.urolonc.2013.05.004.

16. Ozyalvacli G, Ozyalvacli ME, Astarci HM, Boran C, Yesil C, Uyeturk U, Aktas G. Evaluation of different p16 immunostaining methods and the prognostic role of $\mathrm{p} 16 /$ Ki-67 combined expression in non-muscle invasive bladder cancers. Pol J Pathol. 2015; 66:57-66.

17. Poyet C, Buser L, Roudnicky F, Detmar M, Hermanns T, Mannhard D, Hohn A, Ruschoff J, Zhong Q, Sulser T, Moch H, Wild PJ. Connexin 43 expression predicts poor progression-free survival in patients with non-muscle invasive urothelial bladder cancer. J Clin Pathol. 2015; 68:819-24. https://doi.org/10.1136/jclinpath-2015-202898.

18. Bertz S, Otto W, Denzinger S, Wieland WF, Burger M, Stohr R, Link S, Hofstadter F, Hartmann A. Combination of CK20 and Ki-67 immunostaining analysis predicts recurrence, progression, and cancer-specific survival in pT1 urothelial bladder cancer. Eur Urol. 2014; 65:218-26. https://doi.org/10.1016/j.eururo.2012.05.033.

19. Moher D, Liberati A, Tetzlaff J, Altman DG, PRISMA Group. Preferred reporting items for systematic reviews and meta-analyses: the PRISMA statement. PLoS Med. 2009; 6:e1000097. https://doi.org/10.1371/journal.pmed.1000097.

20. Asakura T, Takano Y, Iki M, Suwa Y, Noguchi S, Kubota Y, Masuda M. Prognostic value of Ki-67 for recurrence and progression of superficial bladder cancer. J Urol. 1997; 158:385-8.

21. Lee E, Park I, Lee C. Prognostic markers of intravesical bacillus Calmette-Guerin therapy for multiple, high-grade, stage T1 bladder cancers. Int J Urol. 1997; 4:552-6.

22. Pfister C, Moore L, Allard P, Larue H, Lacombe L, Tetu B, Meyer F, Fradet Y. Predictive value of cell cycle markers p53, MDM2, p21, and Ki-67 in superficial bladder tumor recurrence. Clin Cancer Res. 1999; 5:4079-84.

23. Tomobe M, Shimazui T, Uchida K, Hinotsu S, Akaza H. Argyrophilic nucleolar organizer region in proliferating cell has a predictive value for local recurrence in superficial bladder tumor. J Urol. 1999; 162:63-8. https://doi. org/10.1097/00005392-199907000-00016.

24. Wu TT, Chen JH, Lee YH, Huang JK. The role of bcl-2, p53, and ki-67 index in predicting tumor recurrence for low grade superficial transitional cell bladder carcinoma. J Urol. 2000; 163:758-60.

25. Blanchet P, Droupy S, Eschwege P, Viellefond A, Paradis V, Pichon MF, Jardin A, Benoit G. Prospective evaluation of Ki-67 labeling in predicting the recurrence and progression of superficial bladder transitional cell carcinoma. Eur Urol. 2001; 40:169-75. https://doi.org/49768.

26. Kamai T, Takagi K, Asami H, Ito Y, Oshima H, Yoshida KI. Decreasing of p27(Kip1)and cyclin E protein levels is associated with progression from superficial into invasive bladder cancer. Br J Cancer. 2001; 84:1242-51. https://doi. org/10.1054/bjoc.2000.1736. 
27. Kilicli-Camur N, Kilicaslan I, Gulluoglu MG, Esen T, Uysal $\mathrm{V}$. Impact of p53 and $\mathrm{Ki}-67$ in predicting recurrence and progression of superficial (pTa and pT1) urothelial cell carcinomas of urinary bladder. Pathol Int. 2002; 52:463-9.

28. Sgambato A, Migaldi M, Faraglia B, De Aloysio G, Ferrari P, Ardito R, De Gaetani C, Capelli G, Cittadini A, Trentini GP. Cyclin D1 expression in papillary superficial bladder cancer: its association with other cell cycle-associated proteins, cell proliferation and clinical outcome. Int J Cancer. 2002; 97:671-8.

29. Yan Y, Andriole GL, Humphrey PA, Kibel AS. Patterns of multiple recurrences of superficial (Ta/T1) transitional cell carcinoma of bladder and effects of clinicopathologic and biochemical factors. Cancer. 2002; 95:1239-46. https://doi. org/10.1002/cncr.10822.

30. Dybowski B, Kupryjanczyk J, Rembiszewska A, Pykalo R, Borkowski A. P27(Kip1) and Ki-67 expression analysis in transitional cell carcinoma of the bladder. Urol Res. 2003; 31:397-401. https://doi.org/10.1007/s00240-003-0356-8.

31. Santos L, Amaro T, Costa C, Pereira S, Bento MJ, Lopes P, Oliveira J, Criado B, Lopes C. Ki-67 index enhances the prognostic accuracy of the urothelial superficial bladder carcinoma risk group classification. Int J Cancer. 2003; 105:267-72. https://doi.org/10.1002/ijc.11049.

32. Su JS, Arima K, Hasegawa M, Franco OE, Yanagawa M, Sugimura Y, Kawamura J. Proliferative status is a risk index for recurrence in primary superficial $(\mathrm{pTa} / \mathrm{T} 1)$ low-grade urothelial bladder carcinoma. Hinyokika Kiyo. 2003; 49:649-58.

33. Mhawech P, Greloz V, Oppikofer C, Szalay-Quinodoz I, Herrmann F. Expression of cell cycle proteins in T1a and $\mathrm{T} 1 \mathrm{~b}$ urothelial bladder carcinoma and their value in predicting tumor progression. Cancer. 2004; 100:2367-75. https://doi.org/10.1002/cncr.20306.

34. Kruger S, Mahnken A, Kausch I, Feller AC. P16 immunoreactivity is an independent predictor of tumor progression in minimally invasive urothelial bladder carcinoma. Eur Urol. 2005; 47:463-7. https://doi. org/10.1016/j.eururo.2004.12.018.

35. Theodoropoulos VE, Lazaris AC, Kastriotis I, Spiliadi C, Theodoropoulos GE, Tsoukala V, Patsouris E, Sofras F. Evaluation of hypoxia-inducible factor 1alpha overexpression as a predictor of tumour recurrence and progression in superficial urothelial bladder carcinoma. BJU Int. 2005; 95:425-31. https://doi.org/10.1111/j.1464410X.2005.05314.x.

36. Gonzalez-Campora R, Davalos-Casanova G, Beato-Moreno A, Luque RJ, Alvarez-Kindelan J, Requena MJ, Montironi R, Lopez-Beltran A. Apoptotic and proliferation indexes in primary superficial bladder tumors. Cancer Lett. 2006; 242:266-72. https://doi.org/10.1016/j.canlet.2005.11.006.

37. Quintero A, Alvarez-Kindelan J, Luque RJ, GonzalezCampora R, Requena MJ, Montironi R, Lopez-Beltran A. Ki-67 MIB1 labelling index and the prognosis of primary TaT1 urothelial cell carcinoma of the bladder. J Clin Pathol. 2006; 59:83-8. https://doi.org/10.1136/jcp.2004.022939.
38. Yin H, He Q, Li T, Leong AS. Cytokeratin 20 and Ki-67 to distinguish carcinoma in situ from flat non-neoplastic urothelium. Appl Immunohistochem Mol Morphol. 2006; 14:260-5.

39. Maeng YH, Eun SY, Huh JS. Expression of fibroblast growth factor receptor 3 in the recurrence of non-muscleinvasive urothelial carcinoma of the bladder. Korean J Urol. 2010; 51:94-100. https://doi.org/10.4111/kju.2010.51.2.94.

40. Miyake M, Fujimoto K, Anai S, Ohnishi S, Nakai Y, Inoue T, Matsumura Y, Tomioka A, Ikeda T, Tanaka N, Hirao Y. Clinical significance of heme oxygenase-1 expression in non-muscle-invasive bladder cancer. Urol Int. 2010; 85:355-63. https://doi.org/10.1159/000317785.

41. Seo HK, Cho KS, Chung J, Joung JY, Park WS, Chung MK, Lee KH. Prognostic value of p53 and Ki-67 expression in intermediate-risk patients with nonmuscle-invasive bladder cancer receiving adjuvant intravesical mitomycin $\mathrm{C}$ therapy. Urology. 2010; 76:512.e1-7. https://doi.org/10.1016/j. urology.2010.04.040.

42. Behnsawy HM, Miyake H, Abdalla MA, Sayed MA, Ahmed Ael F, Fujisawa M. Expression of cell cycle-associated proteins in non-muscle-invasive bladder cancer: correlation with intravesical recurrence following transurethral resection. Urol Oncol. 2011; 29:495-501. https://doi. org/10.1016/j.urolonc.2009.08.002.

43. Wosnitzer MS, Domingo-Domenech J, Castillo-Martin M, Ritch C, Mansukhani M, Petrylack DP, Benson MC, McKiernan JM, Cordon-Cardo C. Predictive value of microtubule associated proteins tau and stathmin in patients with nonmuscle invasive bladder cancer receiving adjuvant intravesical taxane therapy. J Urol. 2011; 186:2094-100. https://doi.org/10.1016/j.juro.2011.06.051.

44. Ogata DC, Marcondes CA, Tuon FF, Busato WF Jr, Cavalli G, Czeczko LE. Superficial papillary urothelial neoplasms of the bladder (PTA E PT1): correlation of expression of P53, KI-67 and CK20 with histologic grade, recurrence and tumor progression. Rev Col Bras Cir. 2012; 39:394-400.

45. Oderda M, Ricceri F, Pisano F, Fiorito C, Gurioli A, Casetta G, Zitella A, Pacchioni D, Gontero P. Prognostic factors including Ki-67 and p53 in Bacillus CalmetteGuerin-treated non-muscle-invasive bladder cancer: a prospective study. Urol Int. 2013; 90:184-90. https://doi. org/10.1159/000343431.

46. Okazoe H, Zhang X, Liu D, Shibuya S, Ueda N, Sugimoto M, Kakehi Y. Expression and role of GPR87 in urothelial carcinoma of the bladder. Int J Mol Sci. 2013; 14:12367-79. https://doi.org/10.3390/ijms140612367.

47. Park J, Song C, Shin E, Hong JH, Kim CS, Ahn H. Do molecular biomarkers have prognostic value in primary T1G3 bladder cancer treated with bacillus Calmette-Guerin intravesical therapy? Urol Oncol. 2013; 31:849-56. https:// doi.org/10.1016/j.urolonc.2011.06.004.

48. Ruan J, Wei B, Xu Z, Yang S, Zhou Y, Yu M, Liang J, Jin K, Huang X, Lu P, Cheng H. Predictive value of Sox2 expression in transurethral resection specimens in patients 
with T1 bladder cancer. Med Oncol. 2013; 30:445. https:// doi.org/10.1007/s12032-012-0445-z.

49. Mangrud OM, Gudlaugsson E, Skaland I, Tasdemir I, Dalen I, van Diermen B, Baak JP, Janssen EA. Prognostic comparison of proliferation markers and World Health Organization 1973/2004 grades in urothelial carcinomas of the urinary bladder. Hum Pathol. 2014; 45:1496-503. https://doi.org/10.1016/j.humpath.2014.03.001.

50. Pan $\mathrm{CC}, \mathrm{Yu} \mathrm{HJ}$, Chang $\mathrm{YH}$. The prognostic value of combined clinicopathological and biomarker modelling for non-muscle-invasive bladder cancer. Histopathology. 2014; 65:207-15.

51. Altman DG, Riley RD. Primer: an evidence-based approach to prognostic markers. Nat Clin Pract Oncol. 2005; 2:46672. https://doi.org/10.1038/ncponc0287.

52. McShane LM, Altman DG, Sauerbrei W, Taube SE, Gion M, Clark GM, Statistics Subcommittee of the NCIEWGoCD. REporting recommendations for tumour MARKer prognostic studies (REMARK). Br J Cancer. 2005; 93:387-91. https://doi.org/10.1038/sj.bjc.6602678.
53. Parmar MK, Torri V, Stewart L. Extracting summary statistics to perform meta-analyses of the published literature for survival endpoints. Stat Med. 1998; 17:2815-34.

54. Williamson PR, Smith CT, Hutton JL, Marson AG. Aggregate data meta-analysis with time-to-event outcomes. Stat Med. 2002; 21:3337-51. https://doi.org/10.1002/ sim. 1303 .

55. Tierney JF, Stewart LA, Ghersi D, Burdett S, Sydes MR. Practical methods for incorporating summary time-to-event data into meta-analysis. Trials. 2007; 8:16. https://doi. org/10.1186/1745-6215-8-16.

56. DerSimonian R, Laird N. Meta-analysis in clinical trials. Control Clin Trials. 1986; 7:177-88.

57. Higgins JP, Thompson SG, Deeks JJ, Altman DG. Measuring inconsistency in meta-analyses. BMJ. 2003; 327:557-60. https://doi.org/10.1136/bmj.327.7414.557.

58. Begg CB, Mazumdar M. Operating characteristics of a rank correlation test for publication bias. Biometrics. 1994; 50:1088-101. 